\title{
Needling vs. Kürettage
}

Was das so genannte Needling bei Plantarwarzen bringt, hat ein Team aus Großbritannien in einer randomisierten Studie [1] untersucht. Das Ergebnis ist ernüchternd: Die Methode war nicht effektiver als eine simple Kürettage.

Dornwarzen an der Fußsohle tun weh, sind unschön und auch noch ansteckend. Die herkömmlichen Salizylsäurepräparate zeigen oft erst nach wochen- oder gar monatelanger Anwendung Erfolg, der dann oft nicht von Dauer ist, und auch bei der Kryotherapie kann man langfristig für nichts garantieren.

Eine alternative Methode ist das Needling. Dieses funktioniert im Fall der Warzenbehandlung so: Mit einer Hohlnadel wird wiederholt in das verhornte Gewebe gestochen, und zwar so tief, dass das Instrument bis in die darunterliegende Dermis und ins subkutane Fettgewebe vordringt. Dahinter steckt die Idee, durch die Mikrotraumata eine lokale Entzündung und infolgedessen eine Immunantwort hervorzurufen, die die Läsion zur Abheilung bringen soll.

28 erwachsene Patienten mit Verrucae plantares wurden in einer britischen Fußklinik mit dieser Methode behandelt. Nach einer initialen Kürettierung erfolgten die Punktionen mit einer G-21-Kanüle, wobei die Warze über ihre ganze Ausdehnung "genadelt" wurde. Diesen Patienten hatte man in einem randomisierten Design 25 Kontrollpatienten gegenübergestellt, bei denen lediglich die überschüssige Hornhaut oberflächlich abgetragen wurde.

\section{Jeweils vier Patienten warzenfrei}

Bei der Auswertung nach zwölf Wochen waren in den beiden Gruppen jeweils vier Patienten warzenfrei. Wie Farina Hashmi und Kollegen von der University Salford berichten, entspricht das Erfolgsraten von $14 \%$ für das Needling und von $16 \%$ für das Débridement - kein signifikanter Unterschied also.
Worin die Gruppen sich deutlich unterschieden, war die Schmerzstärke, gemessen mithilfe einer visuellen Analogskala: So berichtete die NeedlingGruppe sowohl vor der Behandlung als auch am Tag danach über deutlich mehr Schmerzen als die Vergleichspatienten (44 gegenüber 24 Punkten vor der Therapiesitzung, 30 vs. 9 Punkte am Tag danach). Insgesamt fünfzehn Patienten - sie stammten alle aus der Nadelgruppe - gaben an, nach der Behandlung ein Schmerzmittel gebraucht zu haben.

\section{Genadelte Patienten zufriedener}

Nichtsdestotrotz zeigten sich zwölf Wochen nach der Sitzung $82 \%$ der genadelten Patienten bereit, den Eingriff zu wiederholen (in der Kontrollgruppe waren es mit $60 \%$ signifikant weniger), und auch in puncto Zufriedenheit lagen erstere deutlich vorn.

Eine Needling-Intervention kostete im Schnitt gut 14 Pfund mehr als das Débridement. Einen objektiven Nutzen, so das Fazit der Autoren, habe die Methode jedenfalls nicht erbracht.

\section{Literatur \\ 1. Hashmi F et al (2017) The EVerT2 (Effective Verruca Treatments 2) trial: a randomized controlled trial of needling vs. nonsur- gical debridement for the treatment of plantar verrucae. Br J Dermatol. https:// doi.org/10.1111/bjd.15751}

Quelle: Dr. Elke Oberhofer, SpringerMedizin.de

Hier steht eine Anzeige. Springer 